\title{
Repositioning compounds from cancer drug discovery to IPF: PI3K inhibition
}

\author{
Claudia A Staab-Weijnitz, Oliver Eickelberg
}

Idiopathic pulmonary fibrosis (IPF) is a fatal interstitial lung disease that presents with a 5-year survival of $20-30 \%$, hence with a mortality rate as high as many aggressive cancer types. ${ }^{1}$ According to a current analysis by the British Lung Foundation, the incidence of IPF in the UK has long been underestimated (https:// www.blf.org.uk/). In addition, a recent systematic review shows that IPF incidence rates have increased worldwide. ${ }^{2}$ Two recently approved treatments for IPF, pirfenidone and nintedanib, have been shown to decelerate lung function decline in IPF, but do not halt disease progression or cure the disease. ${ }^{3}{ }^{4}$ Hence, there is a clear need for novel therapeutic strategies, which manifests in (at time of writing) 46 registered interventional clinical studies for IPF (http://clinicaltrials. gov) and a continued quest for novel drug targets by the scientific community, fuelled by an increased understanding of IPF pathogenesis. ${ }^{5}$ Considering the substantial pathological heterogeneity of IPF and the multitude of dysregulated signalling pathways, ${ }^{6}$ it is likely that a successful cure of IPF will involve not a single, but several active agents, specifically targeting different pathomechanistic aspects of IPF.

With de novo drug development being an expensive and time-consuming process, it seems highly sensible to examine whether effective treatment for other disease entities with significant pathomechanistic overlap with IPF is suitable for IPF therapy as well. Notably, repositioning of drugs can shorten drug development time by $>50 \%$ as phases like chemical optimisation, safety assessment or pharmacokinetic (PK) profiling can be bypassed. ${ }^{7}$ In this context, several recent studies and reviews have highlighted similarities between IPF and cancer, including genetic and epigenetic changes, activation of migration and invasion, altered

Comprehensive Pneumology Center, University Hospital, Ludwig-Maximilians-University and Helmholtz Zentrum München, Munich, Germany

Correspondence to Professor Oliver Eickelberg, Comprehensive Pneumology Center, University Hospital, Ludwig-Maximilians-University and Helmholtz Zentrum München, Munich 81377, Germany;

oliver.eickelberg@helmholtz-muenchen.de responses to growth stimuli or suppressors, an inflammatory component and dysregulated signalling pathways. $^{8-11}$ Notably, nintedanib was originally developed for anticancer treatment and has proven effectiveness in several oncology clinical trials prior to its approval for IPF therapy, clearly supporting this concept. ${ }^{12}$ Wouldn't it be reasonable, therefore, to evaluate potential overlaps between cancer and IPF and choose novel approaches for therapeutic intervention based on such similarities?

Mercer et $\mathrm{l}^{13}$ propose the novel antitumour agent GSK2126458, a pan-class I phosphoinositide 3-kinase (PI3K)/mammalian target of rapamycin (mTOR) inhibitor, as a therapeutic agent for IPF. Following activation via tyrosine kinases and/or G protein-coupled receptors, PI3Ks generate the lipid second messenger phosphatidylinositol-3,4,5-trisphosphate $\left(\operatorname{PtdIns}(3,4,5) \mathrm{P}_{3}\right)$, which coordinates localisation of multiple signalling molecules with a Pleckstrin homology domain, most importantly Akt, at the plasma membrane. ${ }^{14}$ The oncogene PIK3CA, which encodes $\mathrm{p} 110 \alpha$, one of the four class I PI3K catalytic subunit isoforms, and the tumour suppressor gene PTEN (encoding the major $\operatorname{PtdIns}(3,4,5) \mathrm{P}_{3}$ phosphatase) are among the most frequently mutated genes in cancer. Accordingly, activation of PI3K represents a central node in oncogenic signalling, which has prompted extensive studies on targeting the PI3K signalling pathway for cancer therapy. ${ }^{15}$ The mTOR, part of the two multiprotein complexes TORC1 and TORC2, constitutes a major player in the PI3K pathway, acting both upstream and downstream of $\mathrm{Akt}^{15}$ Recent reports have provided evidence that PI3K signalling is also activated in IPF and that its inhibition attenuates transforming growth factor- $\beta$-induced fibroblast proliferation and differentiation to myofibroblasts in vitro, ${ }^{16} 17$ as well as pulmonary fibrosis in vivo. ${ }^{18}$

In this context, the authors set out to evaluate the scientific rationale for PI3K/ mTOR inhibition in IPF and test the efficiency of GSK2126458 in cells and precision-cut lung tissues from patients with IPF. Since PK data from a phase I trial of GSK2126458 in subjects with solid tumours or lymphoma
(NCT00972686) were surfacing, the authors carried out a comprehensive set of in vitro inhibition studies in IPF fibroblasts, carefully determining IC50 values for Akt phosphorylation and proliferation. The integration of these two data sets, the PK data from the ongoing trial and the gathered in vitro data using the described functional readouts, is a major strength of this study as it allowed the authors to deduce a dosing framework, which should ensure safe and effective target engagement in the lungs of patients with IPF. Hereby, Mercer et $a l^{13}$ not only paved the way for an IPF proof-of-mechanism study (NCT01725139), but provided a generally valid roadmap for how to evaluate the potential of drug repositioning at the early clinical trial stage, and the authors should be lauded for this effort.

To date, access to lung tissue from patients with IPF is becoming increasingly limited, and as such, monitoring drug pharmacodynamics and treatment efficacy during IPF clinical trials will be a continuous challenge. In this context, the authors suggest the use of cells obtained by bronchoalveolar lavage, which mostly consist of alveolar macrophages, as a relatively easily accessible pharmacodynamic biosensor. ${ }^{13}$ Even if (myo-)fibroblasts and bronchial/alveolar epithelial cells, and not macrophages, represented the main target cell type for IPF pathogenetic research approaches in the past, this appears as a compelling way to assess whether a drug reached the target tissue and affected resident lung cells.

There are four class I PI3K isoforms termed PI3K $\alpha$, PI3K $\beta, \mathrm{PI} 3 \mathrm{~K} \gamma$ and PI3K $\delta$, which are heterodimers consisting of a catalytic subunit $(\mathrm{p} 110 \alpha, \mathrm{p} 110 \beta, \mathrm{p} 110 \gamma$ or $\mathrm{p} 110 \delta$ ) and a regulatory subunit. Targeting all four class I PI3K isoforms in addition to mTOR, GSK2126458 displays relatively broad target specificity. Clearly, this provides both advantages and disadvantages compared with a single-target approach. On the one hand, compounds with broad substrate specificity frequently require higher doses and are more likely to produce unpredictable off-target effects. ${ }^{14}$ Also, from a mechanistic point of view, the use of this inhibitor makes it impossible to dissect the relative contribution of class I PI3K isoforms or mTOR inhibition to the observed effects. On the other hand, it has been reported that class I PI3Ks show considerable functional redundancy, at least in haematopoietic cells, which argues for the use of pan-class I PI3K inhibitors in the context of haematopoietic malignancies. ${ }^{19}$ Also, many patients exhibit IPF and lung cancer and, 
irrespective of disease-specific isoform involvement, in these cases a pan-class I PI3K inhibitor might kill two birds with one stone. ${ }^{20}$ Furthermore, the PI3K-Akt-mTOR pathway encompasses a sophisticated negative feedback system emphasising the potential need to hit more than one node in the network. To date, however, no evidence for functional redundancy of PI3K enzymes in IPF has been reported. In contrast, Conte et $a l^{16}{ }^{17}$ have provided evidence that the isoform $\mathrm{p} 110 \gamma$ plays a central role in fibroblast proliferation and myofibroblast differentiation in IPF. Also, although inhibition of mTOR has been shown to counteract profibrotic gene expression and protect from pulmonary fibrosis, ${ }^{21} 22$ results from clinical trials have provided evidence that mTOR inhibition might actually induce interstitial lung disease. ${ }^{23}$ Hence, the current data do not unequivocally justify the application of a pan-class I $\mathrm{PI} 3 \mathrm{~K} / \mathrm{mTOR}$ inhibitor in isolated IPF, but offer hope that it will help in select cases.

In conclusion, in light of an unabated need for novel IPF treatment options and the recently accumulated evidence for remarkable similarities between lung cancer and IPF pathogenesis, Mercer et al put forward an intriguing novel concept for future drug repositioning from earlystage clinical cancer trials for IPF. Undoubtedly, more studies are warranted to decipher the relative contribution of the different PI3K isoforms and mTOR to IPF pathogenesis, address the possibility of isoform-specific targeting of PI3K for IPF treatment and critically examine the efficacy of mTOR inhibition versus its adverse pulmonary effects.

Competing interests None declared.

Provenance and peer review Commissioned; internally peer reviewed.

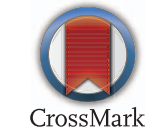

To cite Staab-Weijnitz CA, Eickelberg O. Thorax 2016;71:675-676.

Published Online First 15 June 2016

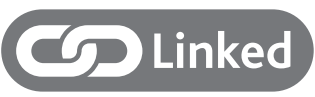

http://dx.doi.org/10.1136/thoraxjnl-2015-207429

Thorax 2016;71:675-676.

doi:10.1136/thoraxjnl-2016-208680

\section{REFERENCES}

1 du Bois RM. An earlier and more confident diagnosis of idiopathic pulmonary fibrosis. Eur Respir Rev 2012;21:141-6.

2 Hutchinson J, Fogarty A, Hubbard R, et al. Global incidence and mortality of idiopathic pulmonary fibrosis: a systematic review. Eur Respir J 2015;46:795-806.

3 King TE Jr, Bradford WZ, Castro-Bernardini S, et al. A phase 3 trial of pirfenidone in patients with idiopathic pulmonary fibrosis. N Engl J Med 2014;370:2083-92.

4 Richeldi L, du Bois RM, Raghu G, et al. Efficacy and safety of nintedanib in idiopathic pulmonary fibrosis. N Engl J Med 2014;370:2071-82.

5 O'Riordan TG, Smith V, Raghu G. Development of novel agents for idiopathic pulmonary fibrosis: progress in target selection and clinical trial design. Chest 2015;148:1083-92.

6 DePianto DJ, Chandriani S, Abbas AR, et al. Heterogeneous gene expression signatures correspond to distinct lung pathologies and biomarkers of disease severity in idiopathic pulmonary fibrosis. Thorax 2015;70:48-56.

7 Ashburn TT, Thor KB. Drug repositioning: identifying and developing new uses for existing drugs. Nat Rev Drug Discov 2004;3:673-83.

8 Horowitz JC, Osterholzer JJ, Marazioti A, et al. "Scar-cinoma": viewing the fibrotic lung mesenchymal cell in the context of cancer biology. Eur Respir J 2016;47:1842-54.

9 Selman M, Pardo A, Kaminski N. Idiopathic pulmonary fibrosis: aberrant recapitulation of developmental programs? PLoS Med 2008;5:e62.
10 Meiners S, Eickelberg 0, Königshoff M. Hallmarks of the ageing lung. Eur Respir J 2015;45:807-27.

11 Königshoff M. Lung cancer in pulmonary fibrosis: tales of epithelial cell plasticity. Respiration 2011;81:353-8.

12 Hilberg F, Roth GJ, Krssak M, et al. BIBF 1120: triple angiokinase inhibitor with sustained receptor blockade and good antitumor efficacy. Cancer Res 2008;68:4774-82

13 Mercer PF, Woodcock HV, Eley JD, et al. Exploration of a potent PI3 kinase/mTOR inhibitor as a novel anti-fibrotic agent in IPF. Thorax 2016;71: 701-11.

14 Vanhaesebroeck B, Guillermet-Guibert J, Graupera $M$, et al. The emerging mechanisms of isoform-specific PI3K signalling. Nat Rev Mol Cell Biol 2010;11:329-41.

15 Fruman DA, Rommel C. PI3K and cancer: lessons, challenges and opportunities. Nat Rev Drug Discov 2014;13:140-56.

16 Conte $\mathrm{E}$, Fruciano $\mathrm{M}$, Fagone $\mathrm{E}$, et al. Inhibition of PI3K prevents the proliferation and differentiation of human lung fibroblasts into myofibroblasts: the role of class I P110 isoforms. PLOS ONE 2011;6: e24663.

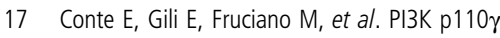
overexpression in idiopathic pulmonary fibrosis lung tissue and fibroblast cells: in vitro effects of its inhibition. Lab Invest 2013;93:566-76.

18 Le Cras TD, Korfhagen TR, Davidson C, et al. Inhibition of PI3K by PX-866 prevents transforming growth factor-alpha-induced pulmonary fibrosis. Am J Pathol 2010;176:679-86.

19 Foukas LC, Berenjeno IM, Gray A, et al. Activity of any class IA PI3K isoform can sustain cell proliferation and survival. Proc Natl Acad Sci USA 2010;107:11381-6.

20 Lee T, Park JY, Lee HY, et al. Lung cancer in patients with idiopathic pulmonary fibrosis: clinical characteristics and impact on survival. Respir Med 2014;108:1549-55.

21 Korfhagen TR, Le Cras TD, Davidson CR, et al. Rapamycin prevents transforming growth factor-alpha-induced pulmonary fibrosis. Am J Respir Cell Mol Biol 2009;41:562-72.

22 Simler NR, Howell DC, Marshall RP, et al. The rapamycin analogue SDZ RAD attenuates bleomycin-induced pulmonary fibrosis in rats. Eur Respir J 2002;19:1124-7.

23 Lopez P, Kohler S, Dimri S. Interstitial lung disease associated with mTOR inhibitors in solid organ transplant recipients: results from a large phase III clinical trial program of everolimus and review of the literature. J Transplant 2014;2014:305931. 